\title{
Influence of rigid boundary on the propagation of torsional surface wave in an inhomogeneous layer
}

\author{
Shishir Gupta*, Rehena Sultana and Santimoy Kundu \\ Department of Applied Mathematics, Indian School of Mines, Dhanbad 826 004, India. \\ ${ }^{*}$ Corresponding author.e-mail: shishir_ism@yahoo.com
}

The present work illustrates a theoretical study on the effect of rigid boundary for the propagation of torsional surface wave in an inhomogeneous crustal layer over an inhomogeneous half space. It is believed that the inhomogeneity in the half space arises due to hyperbolic variation in shear modulus and density whereas the layer has linear variation in shear modulus and density. The dispersion equation has been obtained in a closed form by using Whittaker's function, which shows the variation of phase velocity with corresponding wave number. Numerical results show the dispersion equations, which are discussed and presented by means of graphs. Results in some special cases are also compared with existing solutions available from analytical methods, which show a close resemblance. It is also observed that, for a layer over a homogeneous half space, the velocity of torsional waves does not coincide with that of Love waves in the presence of the rigid boundary, whereas it does at the free boundary. Graphical user interface (GUI) software has been developed using MATLAB 7.5 to generalize the effect of various parameter discussed.

\section{Introduction}

The study of surface waves in half space and layered media are very important to seismologists in understanding the cause and estimation of damage due to earthquakes. Thus, modelling of seismic wave propagation plays a significantly important role and is of great utility in the exploration of petroleum, earthquake disaster prevention, civil engineering, and signal processing. The comprehensive study of propagation of surface waves has already been well documented in Achenbach (1973), Ewing et al. (1957), and Bath (1968). Our globe is a spherical body having finite dimensions and the elastic waves generated must receive the effect of the boundaries. Naturally, this concept leads us to the investigation of boundary waves or surface waves, i.e., the waves which are confined to some surface during their propagation. One such type of surface wave is available in the inhomogeneous earth, known as torsional surface wave. These waves are horizontally polarized but give a twist to the medium when it propagates. Unfortunately, literature available for torsional surface wave propagation is relatively less compared with other surface waves namely, Love, Rayleigh, and Stoneley surface waves. The Earth is considered to be a layered elastic medium with variation in density and rigidity in constitutent layers. Therefore the torsional surface wave must propagate during earthquakes. In a seismogram, some disturbances are observed in between the arrival of Rayleigh and Love wave disturbances. As sufficient information was not available earlier, these disturbances were termed as 'noise' and are ignored in the study of seismic waves. This 'noise' may

Keywords. Torsional surface waves; phase velocity; rigid boundary; hyperbolic; inhomogeneity. 
be due to the torsional waves that propagate in the inhomogeneous earth and the attention of the seismologists may be drawn to this. The Earth's crust contains some hard and soft rocks or materials that may exhibit self-reinforcement property, and inhomogeneity, which is a trivial characteristic of the Earth. These facts motivate us to investigate further on torsional surface wave. Numerous papers on the concerned subject have been published in various journals. In fact the study of surface waves for homogeneous, inhomogeneous, and layerd media has been of central interest to theoritical seismologists. The study of surface waves in a homogeneous half space was initiated by Rayleigh (1885). According to him, the isotropic homogeneous elastic half space does not allow a torsional surface wave to propagate. Later on Meissner (1921) mentioned that in an inhomogeneous elastic half space with quadratic variation of shear modulus and density varying linearly with depth, torsional surface waves do exist. Haskel (1953) studied the dispersion of surface waves in multilayered media. Bhattacharya (1975) investigated the torsional wave propagation in a two layered circular cylinder with imperfect bond. Vardoulakis (1984) has shown that torsional surface waves also propagate in Gibson's half space, where the shear modulus varies linearly with depth but the density remains constant. The two studies by Vrettos (1990a, b) have provided so much insight on the effects of in-homogeneity on surface waves caused by line loads. The propagation of torsional waves in an initially stressed cylinder was discussed by Dey and Dutta (1992).

The study on torsional surface waves got much attention by the beginning of the second quarter of past 20th century. Georgiadis et al. (2000) have examined the torsional surface wave in a gradient elastic half space. The commendable work done by Davini et al. (2008) cannot be overlooked as their contribution towards torsional wave propagation. More recently much work has been published in different journals on the propagation of torsional surface waves. An investigation about the propagation of torsional waves in an inhomogeneous layer over an inhomogeneous half space was proposed by Chattopadhyay et al. (2011), while the propagation of torsional surface waves in heterogeneous anisotropic medium with constant density and variable rigidity was already discussed by Dey et al. (1996). The effect of a rigid boundary on the propagation of a torsional wave, in a homogeneous layer over a heterogeneous half space was proposed by Gupta et al. (2010). The paper has discussed the effect of rigid boundary on the propagation of torsional wave with exponential variation of inhomogeneity in the half-space. Ozturk and Akbarov (2009) have studied torsional wave propagation in a pre-stressed circular cylinder embedded in a pre-stressed elastic medium. Akbarov et al. (2011) have studied torsional wave dispersion in a finitely pre-strained hollow sandwich circular cylinder. The torsional wave propagation in earth's crustal layer under the influence of imperfect interface has been discussed by Vishwakarma et al. (2012). Recently Gupta et al. (2013) investigated the propagation of torsional surface wave in an inhomogeneous layer over an initially stressed inhomogeneous half space of linear type and the study of both compressive and tensile initial stresses have been made.

In this present problem torsional surface wave propagation in an inhomogeneous layer of finite thickness over an inhomogeneous half space has been studied. The inhomogeneity of the layer has been taken as $\mu=\mu_{0}\left(1+m_{0} z\right), \rho=\rho_{0}\left(1+n_{0} z\right)$, where $\mu$ and $\rho$ are the rigidity and mass density respectively, $m_{0}$ and $n_{0}$ are constants having dimensions that are inverse of length. An attempt has been made to investigate the effect of rigid boundary on torsional wave propagation in an inhomogeneous layer over an inhomogeneous half space. In the inhomogeneous crustal layer, density and rigidity vary linearly with depth whereas hyperbolic variation is taken for the half space. Sari and Salk (2002) took the variation in the density of sediments with depth as a hyperbolic function. Bullen (1940) approximated the density as a linear function of depth parameter. In order to reveal the effect of density, rigidity, and inhomogeneity on the propagation of torsional surface wave, numerical computations of dispersion equations have been performed with different values of parameters representing the above charecteristics. It is seen that as the value of density parameter increases, the velocity of torsional surface wave decreases. It has also been found that as the value of rigidity parameter increases, the velocity of torsional surface wave decreases.

\section{Formulation of the problem}

We consider propagation of torsional surface waves in an inhomogeneous layer of finite thickness $\mathrm{H}$ with linear variation in rigidity and density over an inhomogeneous half space with hyperbolic variation in rigidity and density. The top of the layer is assumed to be rigid. For torsional waves, vibrations refer to rotationally symmetric vibrations of an elastic body for which the only particle displacement is oscillatory rotation about the axis of rotational symmetry. This can be concisely phrased in mathematical terms using cylindrical coordinate system with the origin at the source point and the $z$ axis towards the interior of the elastic half-space 
as shown in figure 1 . The following variation in the rigidity and the mass density are taken into account.

For the crustal layer,

$$
\mu=\mu_{0}\left(1+m_{0} z\right), \rho=\rho_{0}\left(1+n_{0} z\right), m_{0}>0, n_{0}>0
$$

where $\mu_{0}, \rho_{0}, \mu_{1}$ and $\rho_{1}$ are constants.

For the half-space,

$$
\begin{aligned}
& \mu=\mu_{1} \cosh ^{2}\left(z / b_{2}\right), \\
& \rho=\rho_{1} \cosh ^{2}\left(z / b_{2}\right), \quad b_{2}>0 .
\end{aligned}
$$

In the above, $\mu$ and $\rho$ are the rigidity and the mass density of the media respectively, where $m_{0}, n_{0}$ are constants having dimensions that are inverse of length and $b_{2}$ has the dimension of length.

\section{Solution}

The dynamical equation of motion is Love (1927)

$$
\left\{\begin{array}{l}
\frac{\partial \sigma_{r r}}{\partial r}+\frac{1}{r} \frac{\partial \sigma_{r \theta}}{\partial \theta}+\frac{\partial \sigma_{r z}}{\partial z}+\frac{\sigma_{r r}-\sigma_{\theta \theta}}{r}=\rho \frac{\partial^{2} u}{\partial t^{2}} \\
\frac{\partial \sigma_{r \theta}}{\partial r}+\frac{1}{r} \frac{\partial \sigma_{\theta \theta}}{\partial \theta}+\frac{\partial \sigma_{\theta z}}{\partial z}+\frac{2 \sigma_{r \theta}}{r}=\rho \frac{\partial^{2} v}{\partial t^{2}} \\
\frac{\partial \sigma_{r z}}{\partial r}+\frac{1}{r} \frac{\partial \sigma_{\theta z}}{\partial \theta}+\frac{\partial \sigma_{z z}}{\partial z}+\frac{\sigma_{r z}}{r}=\rho \frac{\partial^{2} w}{\partial t^{2}}
\end{array}\right.
$$

where $\sigma_{r r}, \sigma_{\theta \theta}, \sigma_{z z}, \sigma_{r z}, \sigma_{r \theta}$ and $\sigma_{\theta z}$ are the respective stress components, and $u, v, w$ are the respective displacement components. Now, the stress-strain relations are given by

$$
\left\{\begin{array}{lll}
\sigma_{r r}=\lambda \Omega+2 \mu e_{r r}, & & \sigma_{\theta \theta}=\lambda \Omega+2 \mu e_{\theta \theta} \\
\sigma_{z z}=\lambda \Omega+2 \mu e_{z z}, & & \sigma_{r \theta}=2 \mu e_{r \theta} \\
\sigma_{r z}=2 \mu e_{r z}, & & \sigma_{\theta z}=2 \mu e_{\theta z}
\end{array}\right.
$$

where $\lambda$ and $\mu$ are Lame's constants, and $\Omega=\left(\frac{\partial u}{\partial r}+\frac{1}{r} \frac{\partial v}{\partial \theta}+\frac{u}{r}+\frac{\partial w}{\partial z}\right)$ denotes the dilatation.

The strain-displacement relations are

$$
\left\{\begin{array}{l}
e_{r r}=\frac{1}{2} \frac{\partial u}{\partial r}, e_{\theta \theta}=\frac{1}{2}\left(\frac{1}{r} \frac{\partial v}{\partial \theta}+\frac{u}{r}\right), e_{z z}=\frac{1}{2} \frac{\partial w}{\partial z} \\
e_{r \theta}=\frac{1}{2}\left(\frac{1}{r} \frac{\partial u}{\partial \theta}+\frac{\partial v}{\partial r}-\frac{v}{r}\right), e_{\theta z}=\frac{1}{2}\left(\frac{\partial v}{\partial z}+\frac{1}{r} \frac{\partial w}{\partial \theta}\right) \\
e_{z r}=\frac{1}{2}\left(\frac{\partial w}{\partial r}+\frac{\partial u}{\partial z}\right)
\end{array}\right.
$$

The torsional wave is characterized by the displacements

$$
u=0, \quad w=0, \quad v=v(r, z, t) .
$$

Now, considering equations (2-4), the dynamical equations of motion for torsional surface waves propagating in the radial direction can be written as:

$$
\frac{\partial \sigma_{r \theta}}{\partial r}+\frac{\partial \sigma_{z \theta}}{\partial z}+\frac{2 \sigma_{r \theta}}{r}=\rho(z) \frac{\partial^{2} v}{\partial t^{2}}
$$

where $v(r, z, t)$ is the displacement along the $\theta$ (azimuthal) direction and $r$ is the radial coordinates. The stress is related to the displacement component by

$$
\sigma_{r \theta}=\mu(z)\left(\frac{\partial v}{\partial r}-\frac{v}{r}\right), \quad \sigma_{z \theta}=\mu(z)\left(\frac{\partial v}{\partial z}\right) .
$$

Using equation (6), equation (5) takes the form

$$
\begin{aligned}
\mu(z) & \left(\frac{\partial^{2}}{\partial r^{2}}+\frac{1}{r} \frac{\partial}{\partial r}-\frac{1}{r^{2}}\right) v+\frac{\partial}{\partial z}\left(\mu(z) \frac{\partial v}{\partial z}\right) \\
= & \rho(z) \frac{\partial^{2} v}{\partial t^{2}} .
\end{aligned}
$$

Harmonic wave solution of equation (7) is of the form

$$
v=V(z) J_{1}(K r) \exp (i \omega t)
$$

where $V(z)$ is the solution of the following equation

$$
\frac{d^{2} V(z)}{d z^{2}}+\frac{\mu^{\prime}(z)}{\mu(z)} \frac{d V(z)}{d z}-K^{2}\left(1-\frac{c^{2}}{c_{s}^{2}}\right) V(z)=0
$$

In the above equation, $c=\omega / K$ is the torsional wave velocity, $c_{s}=\sqrt{\mu / \rho}, \omega$ is the angular frequency, $K$ is the wave number and $J_{1}(K r)$ is the first order Bessel function of first kind.

\subsection{Displacement in upper inhomogeneous layer}

In the inhomogeneous crustal layer, we have:

$$
\mu=\mu_{0}\left(1+m_{0} z\right), \quad \rho=\rho_{0}\left(1+n_{0} z\right) .
$$

Using equation (10) in equation (9), we get

$$
\begin{aligned}
\frac{d^{2} V}{d z^{2}} & +\frac{m_{0}}{1+m_{0} z} \frac{d V}{d z}-K^{2}\left(1-\frac{c^{2}}{c_{0}^{2}} \frac{\left(1+n_{0} z\right)}{\left(1+m_{0} z\right)}\right) V(z) \\
& =0
\end{aligned}
$$

where $c_{0}=\sqrt{\mu_{0} / \rho_{0}}$. 
Now substituting $V(z)=\frac{\phi(z)}{\left(1+m_{0} z\right)^{1 / 2}}$ into equation (11) to eliminate the term $d V / d z$, we have:

$$
\begin{aligned}
& \frac{d^{2} \phi(z)}{d z^{2}}+\left(\frac{m_{0}^{2}}{4\left(1+m_{0} z\right)^{2}}-K^{2}\left(1-\frac{c^{2}}{c_{0}^{2}} \frac{\left(1+n_{0} z\right)}{\left(1+m_{0} z\right)}\right)\right) \\
& \times \phi(z)=0 .
\end{aligned}
$$

Introducing the dimensionless quantities

$$
\gamma=\sqrt{1-\frac{c^{2}}{c_{0}^{2}} \frac{n_{0}}{m_{0}}} \quad \text { and } \quad \eta=\frac{2 \gamma K\left(1+m_{0} z\right)}{m_{0}}
$$

in equation (12), we get:

$$
\begin{gathered}
\frac{d^{2} \phi(\eta)}{d z^{2}}+\left(\frac{1}{4 \eta^{2}}-\frac{1}{4}+\frac{R}{2 \eta}\right) \phi(\eta)=0, \\
R=\frac{\omega^{2}\left(m_{0}-n_{0}\right)}{c_{0}^{2} \gamma m_{0}^{2} K}
\end{gathered}
$$

which is the well known Whittaker's equation (Whittaker and Watson 1990).

The solution of Whittaker's equation (13) is given by

$$
\phi(\eta)=D_{1} W_{\frac{R}{2}, 0}(\eta)+D_{2} W_{-\frac{R}{2}, 0}(-\eta),
$$

where $D_{1}$ and $D_{2}$ are arbitrary constants and $W_{\frac{R}{2}, 0}(\eta), W_{-\frac{R}{2}, 0}(-\eta)$ are the Whittaker function.

Hence, the displacement for the torsional wave in the layer is

$$
\begin{aligned}
v_{0}= & \left(\frac{D_{1} W_{\frac{R}{2}, 0}(\eta)+D_{2} W_{\frac{-R}{2}, 0}(-\eta)}{\left(1+m_{0} z\right)^{\frac{1}{2}}}\right) \\
& \times J_{1}(K r) \exp (i \omega t) .
\end{aligned}
$$

\subsection{Displacement in lower inhomogeneous half space}

In the inhomogeneous half-space, we have:

$$
\mu=\mu_{1} \cosh ^{2}\left(\frac{z}{b_{2}}\right), \quad \rho=\rho_{1} \cosh ^{2}\left(\frac{z}{b_{2}}\right) .
$$

Putting $V=V_{1} / \sqrt{\mu}$ in equation (9) and using equation (15), we get:

$$
V_{1}^{\prime \prime}-m_{1}^{2} V_{1}=0
$$

where

$$
m_{1}=K\left(1+\frac{1}{b_{2}^{2} K^{2}}-\frac{c^{2}}{c_{1}^{2}}\right)^{\frac{1}{2}}
$$

\section{Boundary condition}

The following boundary conditions must be satisfied:

(i) At the free surface $z=-H$, when the upper boundary is assumed to be rigid then

$$
v_{0}=0 \text { at } z=-H \text {. }
$$

(ii) At the interface $z=0$, the continuity of the stress requires that

$$
\mu_{0} \frac{\partial v_{0}}{\partial z}=\mu_{1} \frac{\partial v_{1}}{\partial z} \quad \text { at } z=0 .
$$

(iii) The continuity of the displacement requires that

$$
v_{0}=v_{1} \quad \text { at } z=0 .
$$

(iv) Displacement is bounded as:

$$
\lim _{z \rightarrow \infty} v_{1}(z)=0
$$

where $v_{0}$ and $v_{1}$ are the displacement in the layer and the half-space, respectively.

Therefore, the displacement for the torsional wave in the inhomogeneous half-space using boundary condition (16d) becomes

$$
v_{1}=\frac{G e^{-m_{1} z}}{\sqrt{\mu_{1}} \cosh \left(z / b_{2}\right)} J_{1}(K r) e^{i \omega t} .
$$

Now using the boundary conditions (16b), (16c) and (16a), we get:

$$
\begin{aligned}
& D_{1}\left(\left(-\frac{1}{2}\right)\left(\frac{m_{0}}{K}\right) W_{\frac{R}{2}, 0}\left(\frac{2 \gamma K}{m_{0}}\right)+2 \gamma W_{\frac{R}{2}, 0}^{\prime}\left(\frac{2 \gamma K}{m_{0}}\right)\right) \\
& \quad+D_{2}\left(\left(-\frac{1}{2}\right)\left(\frac{m_{0}}{K}\right) W_{-\frac{R}{2}, 0}\left(-\frac{2 \gamma K}{m_{0}}\right)\right. \\
& \left.\quad-2 \gamma W_{-\frac{R}{2}, 0}^{\prime}\left(-\frac{2 \gamma K}{m_{0}}\right)\right) \\
& \quad-G\left(\frac{\mu_{1}}{\mu_{0}}\right)\left(1+\frac{1}{b_{2}^{2} K^{2}}-\frac{c^{2}}{c_{1}^{2}}\right)^{-1 / 2}=0,
\end{aligned}
$$

where dash over Whittaker function denote differentiation with respect to $z$.

$$
D_{1} W_{\frac{R}{2}, 0}\left(\frac{2 \gamma K}{m_{0}}\right)+D_{2} W_{-\frac{R}{2}, 0}\left(-\frac{2 \gamma K}{m_{0}}\right)-G=0 .
$$

also

$$
\begin{aligned}
& D_{1} W_{\frac{R}{2}, 0}\left\{\frac{2 \gamma K}{m_{0}}\left(1-\frac{m_{0}}{K} K H\right)\right\} \\
& \quad+D_{2} W_{-\frac{R}{2}, 0}\left\{-\frac{2 \gamma K}{m_{0}}\left(1-\frac{m_{0}}{K} K H\right)\right\}=0 .
\end{aligned}
$$


From equations (17), (18) and (19) to eliminate $D_{1}, D_{2}$ and $G$ we have:

$$
\left|\begin{array}{ccc}
W_{\frac{R}{2}, 0}\left(\eta^{\prime}\right) & W_{-\frac{R}{2}, 0}\left(-\eta^{\prime}\right) & 1 \\
\left(-\frac{m_{0}}{2 K}\right) W_{\frac{R}{2}, 0}\left(\eta^{\prime}\right)+2 \gamma W_{\frac{R}{2}, 0}^{\prime}\left(\eta^{\prime}\right) & \left(-\frac{m_{0}}{2 K}\right) W_{-\frac{R}{2}, 0}\left(-\eta^{\prime}\right)-2 \gamma W_{-\frac{R}{2}, 0}^{\prime}\left(-\eta^{\prime}\right) & -\frac{\mu_{1}}{\mu_{0}}\left(1+\frac{1}{b_{2}^{2} K^{2}}-\frac{c^{2}}{c_{1}^{2}}\right)^{\frac{1}{2}} \\
W_{\frac{R}{2}, 0}(\eta) & W_{-\frac{R}{2}, 0}(-\eta) & 0
\end{array}\right|=0 .
$$

where $\eta^{\prime}=\frac{2 \gamma K}{m_{0}}$ and $\eta=\left\{\frac{2 \gamma K}{m_{0}}\left(1-\frac{m_{0}}{K} K H\right)\right\}$.

The expansion of Whittaker function up to linear term is taken as the form

$$
W_{\frac{R}{2}, 0}(\eta)=e^{-\eta / 2} \eta^{\frac{R}{2}}\left\{1-\frac{(R / 2-0.5)^{2}}{1 ! \eta}\right\}
$$

Now expanding the above determinant we get:

$$
\begin{gathered}
\frac{T_{4} \exp (\gamma K H)+T_{3} \exp (-\gamma K H)}{T_{2} \exp (\gamma K H)-T_{1} \exp (-\gamma K H)}+\frac{m_{0}}{2 K \sqrt{1-\frac{c^{2}}{c_{0}^{2}} \frac{n_{0}}{m_{0}}}} \\
=\frac{\mu_{1}}{\mu_{0}} \frac{\sqrt{1+\frac{1}{b_{2}^{2} K^{2}}-\frac{c^{2}}{c_{1}^{2}}}}{\sqrt{1-\frac{c^{2}}{c_{0}^{2}} \frac{n_{0}}{m_{0}}}}
\end{gathered}
$$

where $T_{1}, T_{2}, T_{3}$ and $T_{4}$ are defined in the Appendix.

Equation (20) is the dispersion equation of torsional wave in an inhomogeneous crustal layer over an inhomogeneous half space when the upper boundary plane is assumed to be rigid.

\section{Particular cases}

\subsection{Case 1}

When $b_{2} \rightarrow \infty$, i.e., when the half-space is isotropic homogeneous, then we have from equation (20)

$$
\frac{T_{4} \exp (\gamma K H)+T_{3} \exp (-\gamma K H)}{T_{2} \exp (\gamma K H)-T_{1} \exp (-\gamma K H)}+\frac{m_{0}}{2 K \sqrt{1-\frac{c^{2}}{c_{0}^{2}} \frac{n_{0}}{m_{0}}}}
$$

$$
=\frac{\mu_{1}}{\mu_{0}} \frac{\sqrt{1-\frac{c^{2}}{c_{1}^{2}}}}{\sqrt{1-\frac{c^{2}}{c_{0}^{2}} \frac{n_{0}}{m_{0}}}}
$$

which is the dispersion equation of torsional wave in an inhomogeneous layer over an isotropic homogeneous half space when the upper boundary plane is assumed to be rigid.

\subsection{Case 2}

When $m_{0} \rightarrow 0, n_{0} \rightarrow 0$, i.e., when the layer is isotropic homogeneous, then equation (20) takes the form

$$
\cot \left(K H \sqrt{1-\frac{c^{2}}{c_{0}^{2}}}\right)=\frac{\mu_{1}}{\mu_{0}} \frac{\sqrt{1+\frac{1}{b_{2}^{2} K^{2}}-\frac{c^{2}}{c_{1}^{2}}}}{\sqrt{\frac{c^{2}}{c_{0}^{2}}-1}}
$$

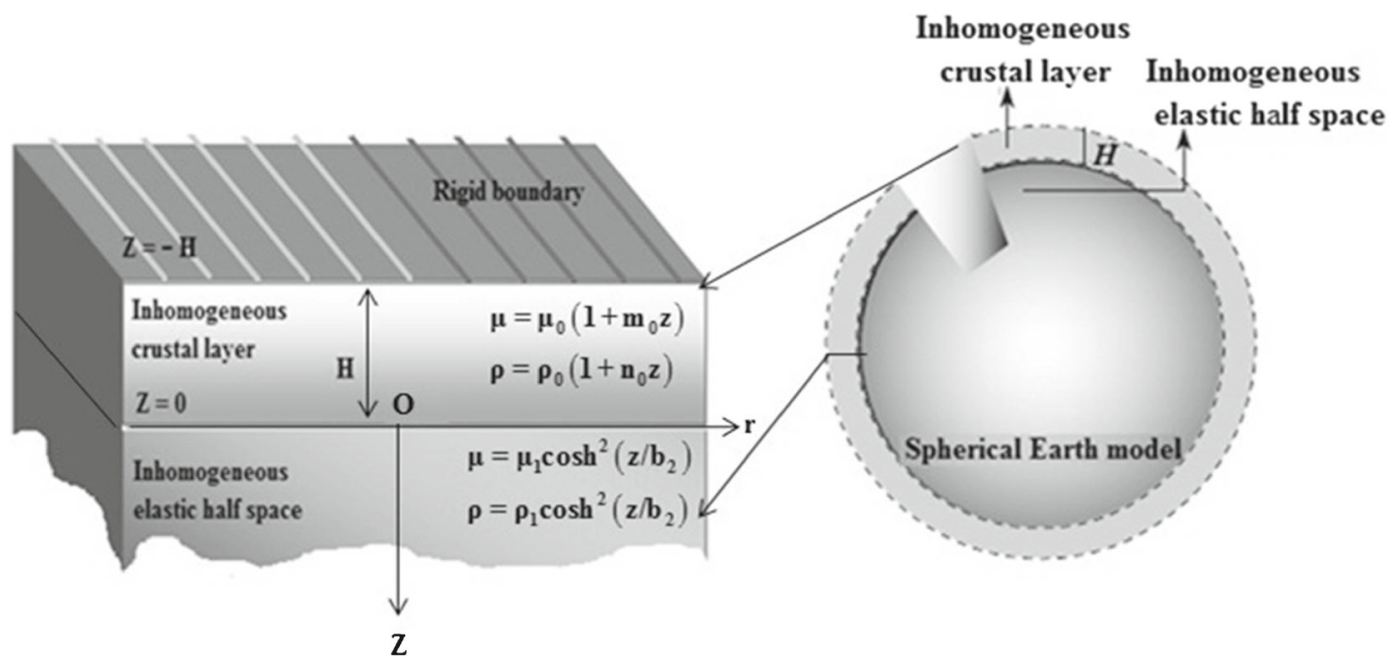

Figure 1. Three-dimensional geometry of the problem. 
which is the dispersion equation of Love type wave in an isotropic homogeneous layer over an inhomogeneous half space when the upper boundary plane is assumed to be rigid (figure 1).

\subsection{Case 3}

When $m_{0} \rightarrow 0, n_{0} \rightarrow 0$, i.e., the layer becomes homogeneous, and the half space becomes homogeneous, i.e., $b_{2} \rightarrow \infty$. The dispersion equation (20) becomes

$$
\cot \left(K H \sqrt{\frac{c^{2}}{c_{0}^{2}}-1}\right)=\frac{\mu_{1}}{\mu_{0}} \frac{\sqrt{1-\frac{c^{2}}{c_{1}}}}{\sqrt{\frac{c^{2}}{c_{0}^{2}}-1}}
$$

which is the dispersion equation of Love type wave in an isotropic homogeneous layer over an isotropic homogeneous half space, when the upper boundary plane is assumed to be rigid. It is interesting to note that in the absence of rigid boundary plane at the top, the classical Love wave equation is

$$
\tan \left(K H \sqrt{\frac{c^{2}}{c_{0}^{2}}-1}\right)=\frac{\mu_{1}}{\mu_{0}} \frac{\sqrt{\left(1-\frac{c^{2}}{c_{1}^{2}}\right)}}{\sqrt{\left(\frac{c^{2}}{c_{0}^{2}}-1\right)}}
$$

\section{Numerical computation and discussion}

In order to interpret the effect of various inhomogeneity parameters $m_{0} / K, n_{0} / K$ on the propagation of torsional surface waves, numerical computation of equations (20) and (23) are performed with different values of elastic constants as given in table 1 (Tierstein 1969; Gubbins 1990).

In figures 2, 3, and 5, curves have been plotted with vertical axis as dimensionless phase velocity $\left(c / c_{0}\right)$ against horizontal axis as dimensionless wave number $(K H)$. Curve 1 represents the classical case of Love wave in all figures. Curves 2,

Table 1. Values of various elastic constants.

\begin{tabular}{lcccccc}
\hline Curves & $\mu_{0}(\mathrm{~N} \cdot \mathrm{m}-2)$ & $\rho_{0}(\mathrm{~kg} \cdot \mathrm{m}-3)$ & $\mu_{1}(\mathrm{~N} \cdot \mathrm{m}-2)$ & $\rho_{1} /(\mathrm{kg} \cdot \mathrm{m}-3)$ & $\mu_{0} / \mu_{1}$ & $\left(c_{0} / c_{1}\right)^{2}$ \\
\hline $1,2,3,4,5$ & $93 \times 10^{9}$ & 7450 & $75.00 \times 10^{9}$ & 4700 & 1.2400 & 0.7823 \\
\hline
\end{tabular}

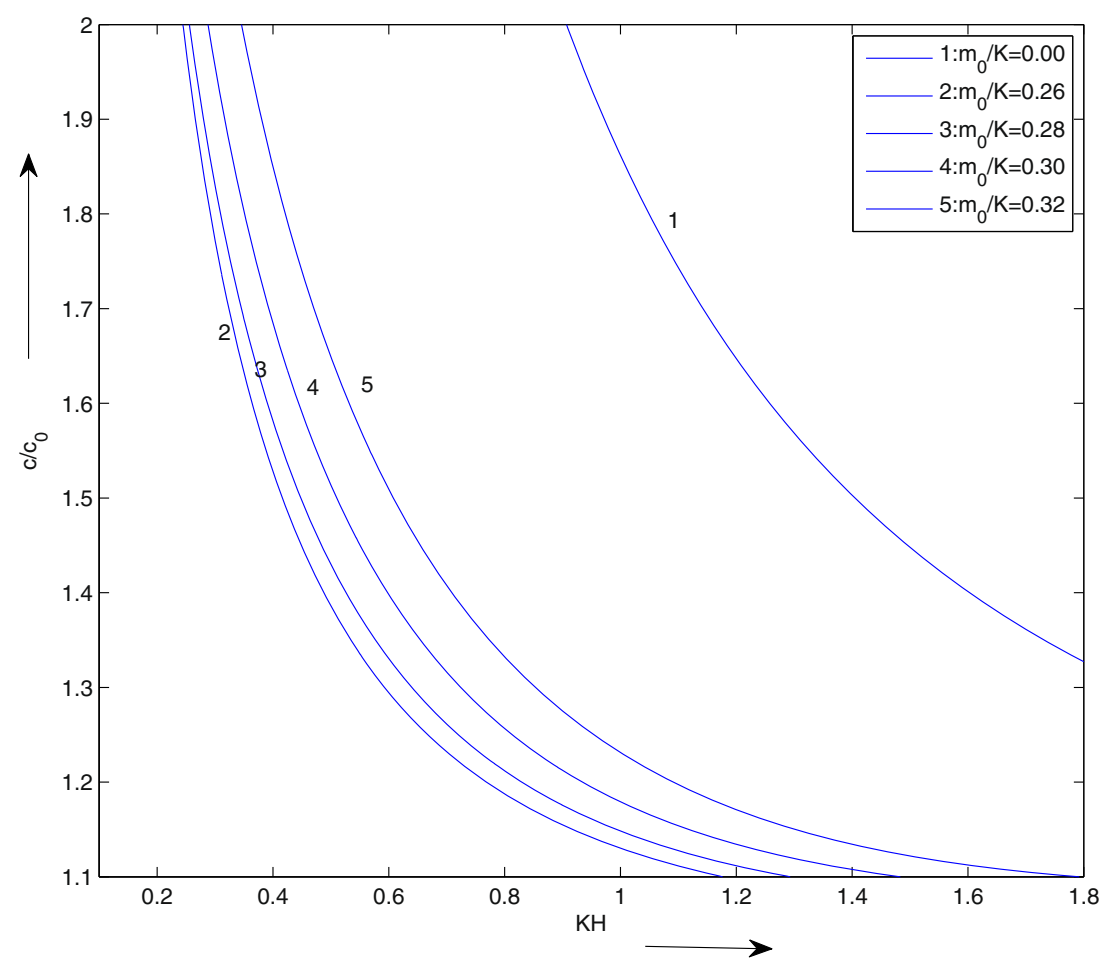

Figure 2. Dimensionless phase velocity against dimensionless wave number demonstrating the influence of inhomogeneity parameter associated with the rigidity of the layer. 
$3,4,5$ in figure 2 are plotted for the various values of inhomogeneity parameter $m_{0} / K$, i.e., 0.26 , $0.28,0.30,0.32$ keeping $n_{0} / K$ constant. Curves 2 , $3,4,5$ in figure 3 are plotted for the various values of $n_{0} / K$, i.e., $0.40,0.42,0.44,0.46$ keeping $m_{0} / K$ constant.

Figure 2 signifies the dispersion curve of the torsional surface wave under the effect of inhomo- geneity parameter $m_{0} / K$ involved in the rigidity of the crustal layer. From this figure we have seen the following:

a) The curve no. 2 of the figure represents that the speed of torsional wave decreases with the increase of wave number. Again we have seen from curve no. 2 to 5 that the dispersion curves

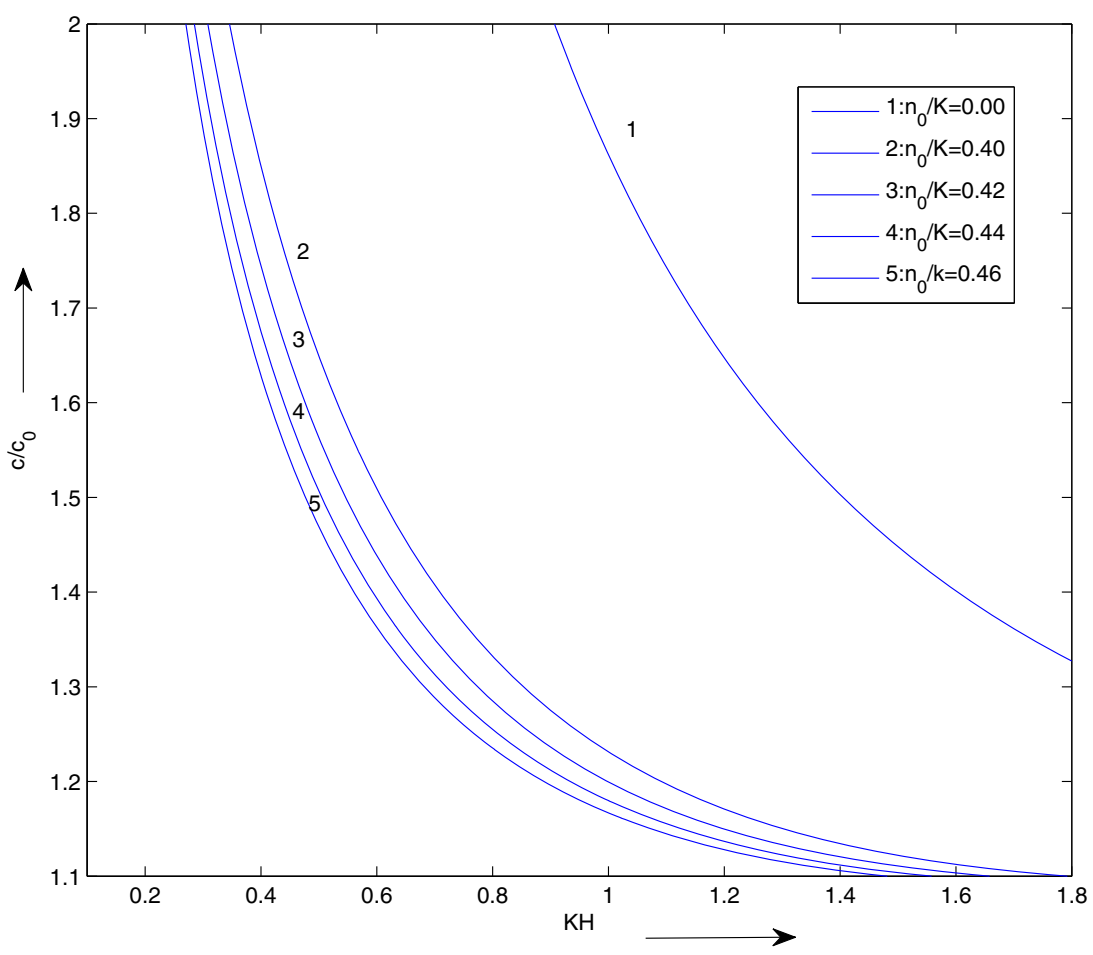

Figure 3. Dimensionless phase velocity against dimensionless wave number demonstrating the influence of inhomogeneity parameter associated with the density of the layer.

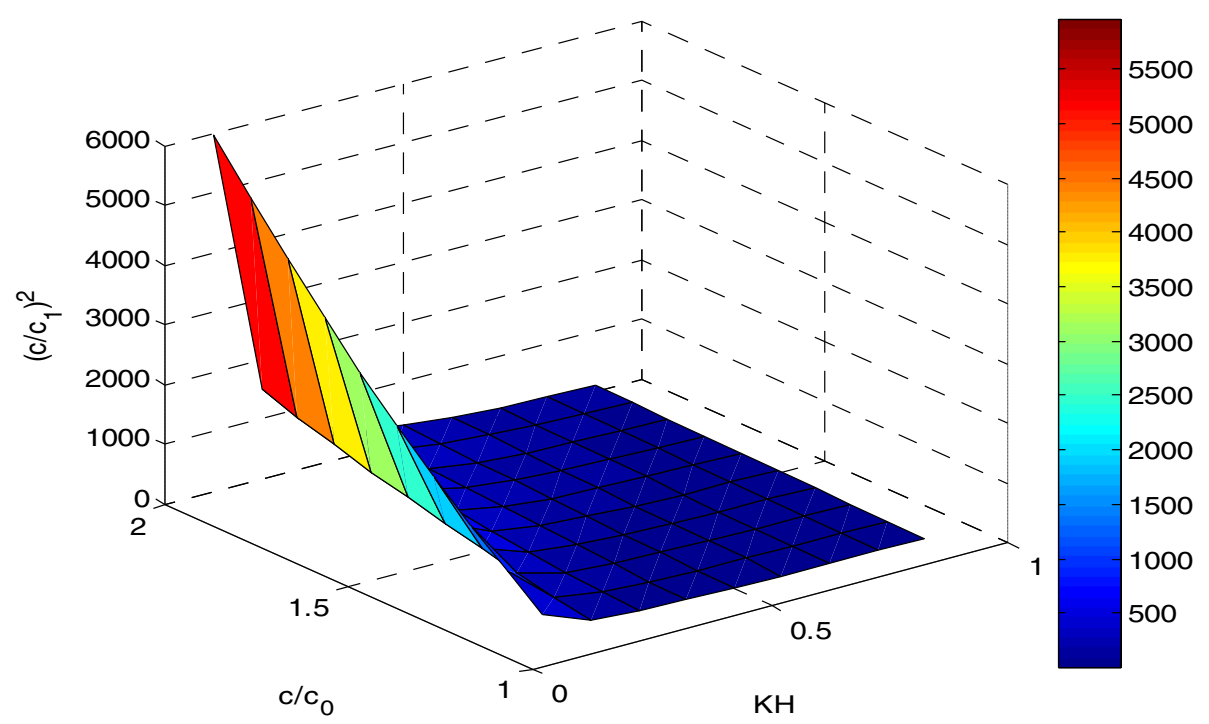

Figure 4. Surface plot of the square of velocity ratio (dimensionless) against dimensionless phase velocity and dimensionless wave number. 


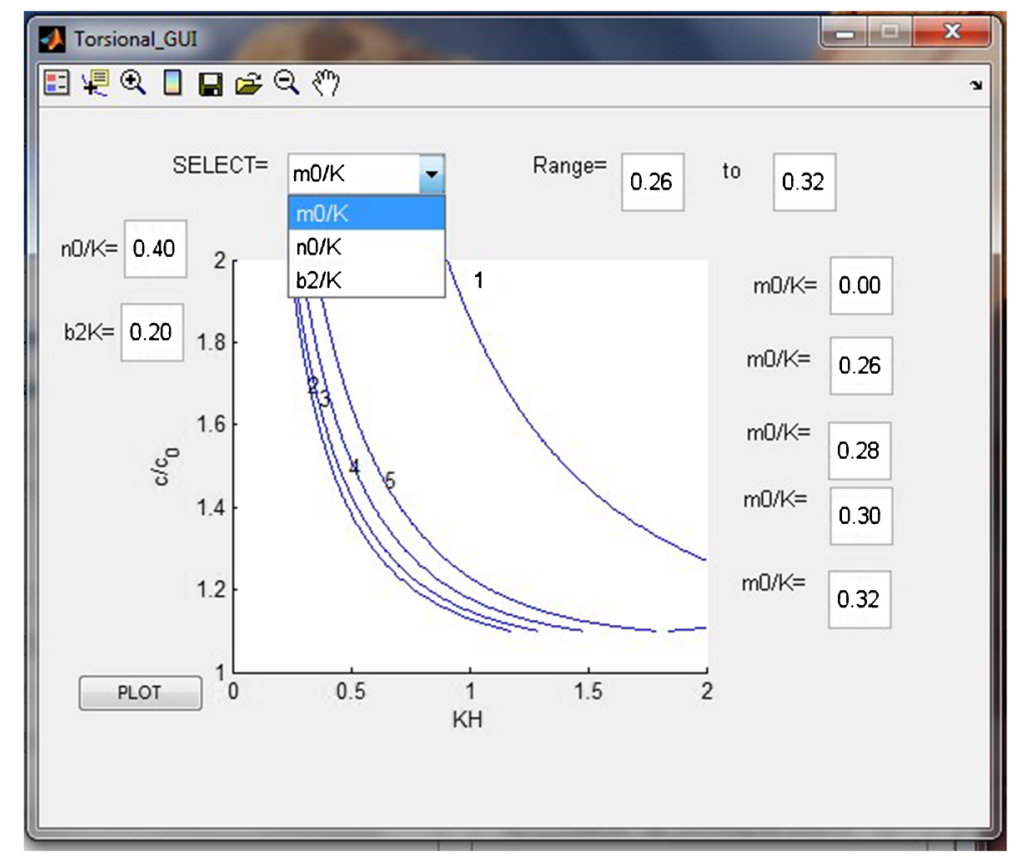

Figure 5. Snapshot of graphical user interface (GUI) developed in MATLAB 7.5.

increase with the increases of inhomogeneity parameter $m_{0} / K$, which justifies that the velocity of the torsional surface wave is directly proportional to the rigidity of the medium.

b) The curve 1 (classical case) is steeper than the curves $2-5$, which reveals that the dimensionless inhomogeneity factor $m_{0} / K$ has a prominent effect on torsional wave propagation.

Figure 3 gives the dispersion curves of torsional surface wave against dimensionless wave number. Our attempt has been to arrive at the effect of inhomogeneity factor $n_{0} / K$. The following effects and observation have been enlisted as:

- The speed of torsional surface wave decreases rapidly with the increase of wave number. For curve nos. 2 to 5 we have seen that dispersion curve decreases with the increase of inhomogeneity parameter $n_{0} / K$.

- The curve 1 (classical case) is steeper than the curves $2-5$, which reveals that the inhomogeneity factor $n_{0} / K$ has a prominent effect on torsional wave propagation.

Keeping in mind the dependence of the square of velocity ratio $\left(c / c_{1}\right)^{2}$ to wave number $K H$ and phase velocity $c / c_{0}$, surface plot of $\left(c / c_{1}\right)^{2}$ (taken along $\mathrm{z}$ axis) against phase velocity (taken along $\mathrm{y}$ axis) and wave number (taken along $\mathrm{x}$ axis) has been shown in figure 4 . This plot shows the combined effect of $K H$ and $\left(c / c_{1}\right)^{2}$ on the phase velocity of torsional surface wave.

Figure 5 represents a screen shot of graphical user interface (GUI) software in MATLAB 7.5 to demonstrate the graph plotted in figure 2 as a sample. This GUI generalizes the finding of the present paper by allowing one to vary the ranges of different dimensionless parameters and also by providing different values to the various parameters involved. This will help one to observe the variations on the phase velocity of torsional surface wave against dimensionless wave number for different sets of values.

\section{Conclusions}

An analytical approach is used to investigate the propagation of torsional surface waves in an inhomogeneous crustal layer over an inhomogeneous elastic half-space when the upper boundary plane is assumed to be rigid. The dispersion equation obtained is in agreement with the result of Love type wave when the inhomogeneity parameters are neglected. Using asymptotic linear expansion of the Whitaker's function we have obtained the dispersion relation for the torsional wave in compact form. Phase velocity is computed numerically and the effect of variation in density and rigidity on the propagation of torsional surface wave is prominent. Effect of various dimensionless elastic parameters and inhomogeneity factor on the dimensionless torsional wave velocity $c / c_{0}$ has been shown graphically. We observed that the dispersion curve of torsional surface wave increases as the inhomogeneity factor $m_{0} / K$ increases, satisfying the fact that the velocity is directly proportional to the rigidity of the medium whereas the dispersion 
curve of torsional surface wave decreases as the inhomogeneity factor $n_{0} / K$ increases, validating the fact that velocity is inversely proportional to the density of the medium. Presentation of surface plot reveals that the velocity ratio $\left(c / c_{1}\right)^{2}$ increases as the value of phase velocity and wave number increases. Graphical user interface software in MATLAB has been developed to generalize the result of figures 3-4 so as to study the effect of various inhomogeneity parameters.

\section{Acknowledgements}

The authors convey their sincere thanks to Indian School of Mines, Dhanbad for providing JRF to Rehena Sultana and also facilitating us with its best facility. Acknowledgement is also due to CSIR, New Delhi for providing financial support through Project No: 25(0227)/13/EMR-II, Project title: "Study of torsional wave in anisotropic and nonhomogeneous media".

\section{Appendix}

$$
\begin{aligned}
& T_{1}=\left(\frac{\eta^{\prime}}{\eta}\right)^{\frac{R}{2}}(-1)^{-\frac{R}{2}}\left(1+\frac{\left(-\frac{R}{2}-\frac{1}{2}\right)^{2}}{\eta}\right) \\
& \times\left(1-\frac{\left(\frac{R}{2}-\frac{1}{2}\right)^{2}}{\eta^{\prime}}\right) \\
& T_{2}=\left(\frac{\eta}{\eta^{\prime}}\right)^{\frac{R}{2}}(-1)^{-\frac{R}{2}}\left(1-\frac{\left(\frac{R}{2}-\frac{1}{2}\right)^{2}}{\eta}\right) \\
& \times\left(1+\frac{\left(-\frac{R}{2}-\frac{1}{2}\right)^{2}}{\eta^{\prime}}\right) \\
& T_{3}=\left(\frac{\eta^{\prime}}{\eta}\right)^{\frac{R}{2}}(-1)^{-\frac{R}{2}}\left(1+\frac{\left(-\frac{R}{2}-\frac{1}{2}\right)^{2}}{\eta}\right) \\
& \left(-1+\frac{R}{\eta^{\prime}}+\frac{1}{\eta^{\prime}}\left(\frac{R}{2}-\frac{1}{2}\right)^{2}\right. \\
& \left.-R \frac{\left(\frac{R}{2}-\frac{1}{2}\right)^{2}}{\eta^{\prime 2}}+\frac{\left(\frac{R}{2}-\frac{1}{2}\right)^{2}}{\eta^{\prime 2}}\right) \\
& T_{4}=\left(\frac{\eta}{\eta^{\prime}}\right)^{\frac{R}{2}}(-1)^{-\frac{R}{2}}\left(1-\frac{\left(\frac{R}{2}-\frac{1}{2}\right)^{2}}{\eta}\right) \\
& \left(-1+\frac{R}{\eta^{\prime}}-\frac{1}{\eta^{\prime}}\left(-\frac{R}{2}-\frac{1}{2}\right)^{2}+R \frac{\left(-\frac{R}{2}-\frac{1}{2}\right)^{2}}{\eta^{\prime 2}}\right. \\
& \left.+\frac{\left(-\frac{R}{2}-\frac{1}{2}\right)^{2}}{\eta^{\prime 2}}\right)
\end{aligned}
$$

\section{References}

Achenbach J D 1973 Wave propagation in elastic solids; New York, North-Holland Publishing Company.

Akbarov S D, Kepceler T and Mert Egilmez M 2011 Torsional wave dispersion in a finitely pre-strained hollow sandwich circular cylinder; J. Sound and Vibration 330 4519-4537.

Bath M 1968 Mathematical Aspects of Seismology; New York, Elsevier Publishing Company.

Bhattacharya R C 1975 On the torsional wave propagation in a two layered circular cylinder with imperfect bonding; Proc. Indian Nat. Sci. Acad. 41(6) 613619.

Bullen K E 1940 The problem of the earth's density variation; Bull. Seismol. Soc. Am. 30(3) 235-250.

Chattopadhyay A, Gupta S, Kumari P and Sharma V K 2011 Propagation of torsional waves in an inhomogeneous layer over an inhomoogeneous half-space; Mecanica 46(4) 671-680.

Davini C, Paroni R and Puntle E 2008 An asymptotic approach to the torsional problem in thin rectangular domains; Meccanica 43(4) 429-435.

Dey S and Dutta A 1992 Torsional wave propagation in an initially stressed cylinder; Proc. Indian Nat. Sci. Acad. 58(5) 425-429.

Dey S, Gupta A K and Gupta S 1996 Torsional surface waves in non-homogeneous and anisotropic medium; J. Acoust. Soc. Am. 99(5) 2737-2741.

Ewing W M, Jardetzky W S and Press F 1957 Elastic waves in Layered media; New York McGraw-Hill.

Georgiadis H G, Vardoulakis I and Lykotrafitis G 2000 Torsional surface waves in a gradient elastic half-space; Wave Motion 31(4) 333-348.

Gubbins D 1990 Seismology and Plate Tectonics; Cambridge University Press, Cambridge.

Gupta S, Chattopadhyay A, Kundu S and Gupta A K 2010 Effect of rigid boundary on the propagation of torsional waves in a homogeneous layer over a heterogeneous halfspace; Arch. Appl. Mech. 80 143-150.

Gupta S, Kundu S and Vishwakarma S K 2013 Propagation of torsional surface wave in an inhomogeneous layer over an initially stressed inhomogeneous half-space; J. Vibration and Control, doi: 10.1177/1077546313493818.

Haskel N A 1953 The dispersion of surface waves on multi-layered media; Bull. Seismol. Soc. Am. 43(1) $17-34$.

Love A E H 1927 The mathematical theory of elasticity; Cambridge University Press, Cambridge.

Meissner E 1921 Elastic surface-waves with dispersion in an inhomogeneous medium; The Quarterly Magazine Naturalist Society in Zurich 66 181-195.

Ozturk A and Akbarov S D 2009 Torsional wave propagation in a pre-stressed circular cylinder embedded in a pre-stressed elastic medium; Appl. Math. Modelling 33 3636-3649.

Rayleigh L 1885 On waves propagated along the plane surface of an elastic solid; Proc. London Math. Soc. 17(3) $4-11$.

Sari C and Salk M 2002 Analysis of gravity anomalies with hyperbolic density contrast: An application to the gravity data of western Anatolia; J. Balkan Geophys. Soc. 5(3) 87-96.

Tierstein H F 1969 Linear Piezoelectric Plate Vibrations; New York, Plenum Press.

Vardoulakis I 1984 Torsional surface waves in inhomogeneous elastic media; Int. J. Numer. Anal. Meth. Geomech. 8 287-296.

Vishwakarma S K, Gupta S and Verma A K 2012 Torsional wave propagation in Earth's crustal layer under the 
influence of imperfect interface; J. Vibration and Control 20(3) 355-369, doi: 10.1177/1077546312461029.

Vrettos C H 1990a In plane vibrations of soil deposits with variable shear modulus II Line load; Int. J. Numer. Anal. Meth. Geomech. 14 649-662.
Vrettos C H 1990b In pulse vibrations of soil deposits with variable shear modulus: I. surface waves; Int. J. Numer. Anal. Meth. Geomech. 14 209-222.

Whittaker E T and Watson G N 1990 A Course in Modern Analysis; Cambridge University Press, Cambridge.

MS received 9 January 2014; revised 8 August 2014; accepted 21 August 2014 